\title{
Not BCL2 mutation but dominant mutation conversation contributed to acquired venetoclax resistance in acute myeloid leukemia
}

Xiang Zhang ${ }^{1,2,3,4,5 \dagger}$, Jiejing Qian ${ }^{1,2+}$, Huafeng Wang ${ }^{1,2,3,4,5 \dagger}$, Yungui Wang ${ }^{1,2 \dagger}$, Yi Zhang ${ }^{1,2}$, Pengxu Qian ${ }^{3,4,5}$, Yinjun Lou ${ }^{1,2}$, Jie Jin ${ }^{1,2,3,4}$ and Honghu Zhu $u^{1,2,3,4,5^{*}}$ (i)

\begin{abstract}
Venetoclax (VEN) plus azacitidine has become the first-line therapy for elderly patients with acute myeloid leukemia (AML), and has a complete remission (CR) plus CR with incomplete recovery of hemogram rate of $\geq 70 \%$. However, the 3 -year survival rate of these patients is $<40 \%$ due to relapse caused by acquired VEN resistance, and this remains the greatest obstacle for the maintenance of long-term remission in VENsensitive patients. The underlying mechanism of acquired VEN resistance in AML remains largely unknown. Therefore, in the current study, nine AML patients with acquired VEN resistance were retrospectively analyzed. Our results showed that the known VEN resistance-associated BCL2 mutation was not present in our cohort, indicating that, in contrast to chronic lymphocytic leukemia, this BCL2 mutation is dispensable for acquired VEN resistance in AML. Instead, we found that reconstructed existing mutations, especially dominant mutation conversion (e.g., expanded FLT3-ITD), rather than newly emerged mutations (e.g., TP53 mutation), mainly contributed to VEN resistance in AML. According to our results, the combination of precise mutational monitoring and advanced interventions with targeted therapy or chemotherapy are potential strategies to prevent and even overcome acquired VEN resistance in AML.
\end{abstract}

Keywords: Venetoclax, Acquired resistance, Acute myeloid leukemia

\footnotetext{
* Correspondence: zhuhhdoc@163.com

${ }^{+}$Xiang Zhang, Jiejing Qian, Huafeng Wang and Yungui Wang contributed equally to this work.

'Department of Hematology, The First Affiliated Hospital, Zhejiang University School of Medicine, \#79 Qingchun Rd, Hangzhou 310003, Zhejiang, China

${ }^{2}$ Key Laboratory of Hematologic Malignancies, Diagnosis and Treatment, Hangzhou, Zhejiang, China

Full list of author information is available at the end of the article
}

(c) The Author(s). 2021 Open Access This article is licensed under a Creative Commons Attribution 4.0 International License, which permits use, sharing, adaptation, distribution and reproduction in any medium or format, as long as you give appropriate credit to the original author(s) and the source, provide a link to the Creative Commons licence, and indicate if changes were made. The images or other third party material in this article are included in the article's Creative Commons licence, unless indicated otherwise in a credit line to the material. If material is not included in the article's Creative Commons licence and your intended use is not permitted by statutory regulation or exceeds the permitted use, you will need to obtain permission directly from the copyright holder. To view a copy of this licence, visit http://creativecommons.org/licenses/by/4.0/ The Creative Commons Public Domain Dedication waiver (http://creativecommons.org/publicdomain/zero/1.0/) applies to the data made available in this article, unless otherwise stated in a credit line to the data. 


\section{To the Editor}

VEN + AZA has become the first-line therapy for elderly patients with $\mathrm{AML}$, and $\mathrm{CR}+\mathrm{CRi}$ rates of $\geq 70 \%$ have been achieved [1, 2]. Despite this, the 3year survival rate of patients who receive VEN + AZA is $<40 \%$, mainly due to acquired VEN-R [3]. However, the underlying mechanisms of VEN-R and the status of $B C L 2^{\text {Mut }}$ in AML, remain largely unknown [4-6].

To address this question, we retrospectively analyzed nine elderly AML patients with acquired VEN-R at our center from July 1, 2018 until June 30, 2020 (Table 1). $B C L 2^{M u t}$ was detected by PCR combined with Sanger sequencing at VEN-I and VEN-R, but no VEN-Rassociated $B C L 2^{M u t}$ was identified (Fig. 1a) [6-9]. Due to the relatively low resolution of Sanger sequencing, these samples were then submitted to TES (Novaseq platform, Illumina), in which 236 recurrently mutated genes in hematological malignancies were included. The average raw sequencing depth on target per sample was $\geq 1000$,

Table 1 Basic characteristics of patients with acquired VEN-R AML in our cohort

\begin{tabular}{ll}
\hline Characteristics & Value \\
\hline Patients (N) & 9 \\
Male/Female (N) & $5 / 4$ \\
Age (year) & 73 (68-78) \\
De novo/Secondary (N) & $8 / 1$ \\
FAB: M0/M1/M4/M5 (N) & $2 / 1 / 3 / 3$ \\
Karyotype: normal/abnormal (N) & $4 / 5$ \\
Bone marrow blast at venetoclax initiation (\%) & $62(23-92)$ \\
Molecular feature at venetoclax initiation (N) & \\
AML1-ETO & 1 \\
NPM1 mutation & 3 \\
FLT3-ITD & 4 \\
DNMT3A mutation & 4 \\
TP53 mutation & 4 \\
ASXL1 mutation & 1 \\
RUNX1 mutation & 2 \\
Bone marrow blast at venetoclax resistance (\%) & 2 \\
Molecular feature at venetoclax resistance (N) & 10.5 (6-74) \\
AML1-ETO & \\
NPM1 mutation & 1 \\
FLT3-ITD & 2 \\
DNMT3A mutation & 3 \\
TP53 mutation & 2 \\
ASXL1 mutation & 1 \\
RUNX1 mutation & 3 \\
Cycles from venetoclax initiation to resistance (N) & \\
\hline
\end{tabular}

and a VAF $\geq 1 \%$ was considered significant. As VEN-Rassociated $B C L 2^{M u t}$ was consistently negative, $B C L 2^{M u t}$ was considered dispensable for acquired VEN-R in AML.

Regarding the difference in the mutational landscape between VEN-I and VEN-R (Supplementary Table 1), the spectrum was skewed in 7/9 patients: $3 / 7$ exhibited a reduction in mutated genes, $1 / 7$ exhibited an increase, and $3 / 7$ showed a reduction in some mutated genes and an increase in others (Fig. 1b). As TP53 mutation has been demonstrated to confer AML VEN-R [10], newly emerged TP53 mutation definitely contributed to VEN$\mathrm{R}$ as shown in Pt \#8 (Fig. 1c). However, newly emerged mutations in the remaining three patients had relatively low VAFs compared to the dominant mutations, which indicated that these mutations existed in sub-clones and played a minor role in acquired VEN-R.

We next addressed the proportion of reconstructed existing mutations. Excluding Pt \#9 without the molecular relapse, $6 / 8$ patients exhibited reconstructed existing mutations, and $4 / 8$ patients showed dominant mutational conversion (Fig. 1d). FLT3-ITD is the most common mutation in AML [11], but whether it affects VEN sensitivity remains controversial [1]. In Pt \#3, \#6, and \#7, the VAF of FLT3-ITD increased, and it had ranged from a minor mutation at VEN-I to the most common mutation at VEN-R (Fig. 1e-g). Although FLT3-ITD was totally absent from Pt \#5, FLT3-ITD still conferred VEN-R for AML in Pt \#3, Pt \#6, and Pt \#7. In Pt \#1, IDH2 $2^{R 140 Q}$ and TP53 ${ }^{L 145 P}$ mutations were the dominant mutations across the entire treatment course; however, their VAFs decreased, while those of $N F 1^{T 419 f s}$ and $P H F 6^{T 300 A}$ mutations gradually increased with AML progression. These findings indicate that minor mutations can expand and possibly contribute to VEN-R (Fig. 1h).

Although VEN-associated $B C L 2^{M u t}$ has been identified in CLL, it was not detected in our AML cohort. There are several possible explanations. First, there was short duration exposure to VEN in AML (AML vs. CLL [months], 5 [3-9] vs. 36[6.5-73]) [12]; second, combination therapy with AZA in AML may have eradicated the emerged $B C L 2^{M u t}$ at an early stage; and third, the standard dose of VEN (400 mg/qd) used in AML patients was not reached in $27 \%$ of CLL patients. Theoretically, $B C L 2^{\text {Mut }}$ may have mediated VEN-R in patients with AML as the duration of exposure increased, but in reality, combination therapy at a standard dose made the possibility of emerged $B C L 2^{\text {Mut }}$ much lower than in CLL. BCL2 $2^{\text {Mut }}$ was still negative in our two cases with $\geq 1$-year exposure duration. In contrast to $B C L 2^{\mathrm{Mut}}$, we found that clonal evolution, including newly emerged mutations and reconstructed existing mutations, mainly contributed to VEN-R in AML. For example, newly emerged TP53 mutation or expanded FLT3-ITD could 


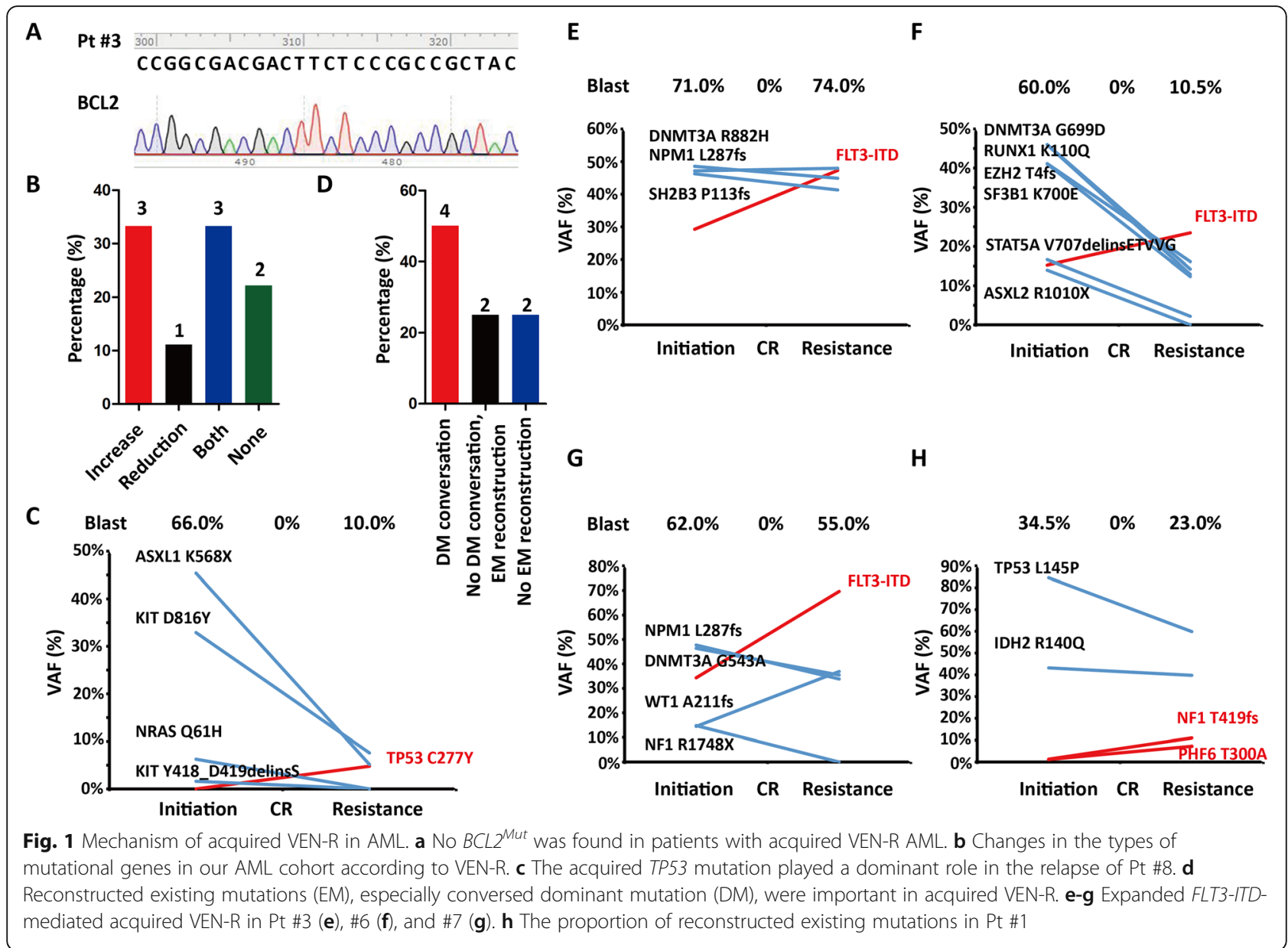

mediate acquired VEN-R in AML, which was also reported by DiNardo [4]. Interestingly, acquired TP53 mutation also mediated VEN-R in CLL independent of $B C L 2^{M u t}$, and it was more common than in AML. Furthermore, reconstructed existing mutations, especially dominant mutation conversion, appear to be more important than newly emerged mutations in acquired VEN-R. More aggressive clinical strategies are required to overcome this mechanism in acquired VEN-R in AML. In our cohort, three patients with AML with expanded FLT3-ITD-mediated acquired VEN-R possibly benefited from dynamic monitoring of FLT3-ITD and early addition of an FLT3 inhibitor to prolong the response to VEN. Therefore, the combination of precise mutational monitoring and advanced interventions with targeted therapy or chemotherapy is key to preventing and overcoming acquired VEN-R in AML.

\section{Abbreviations}

AML: Acute myeloid leukemia; AZA: Azacitidine; BCL2 ${ }^{\text {Mut }}$ : BCL2 mutation; CLL: Chronic lymphocytic leukemia; CR: Complete remission; CRi: Complete remission with incomplete recovery of hemogram; PCR: Polymerase chain reaction; Pt: Patient; TES: Targeted-exome-sequencing; VAF: Variant allele frequency; VEN: Venetoclax; VEN-I: Venetoclax initiation; VEN-R: Venetoclax resistance

\section{Supplementary Information}

The online version contains supplementary material available at https://doi. org/10.1186/s40364-021-00288-7.

Additional file 1: Table S1. Differences in the mutational landscape between patients with VEN-I and VEN-R AML.

\section{Acknowledgements}

We thanked all members of the key laboratory of diagnosis and treatment in hematologic malignancies of Zhejiang and bone marrow morphology laboratory of the First Affiliated Hospital to Zhejiang University College of Medicine for technical support to diagnosis.

\section{Authors' contributions}

H.-H. Z. and X. Z. designed this study. J.-J. Q., H.-F. W., and Y. Z. collected the clinical materials. Y.-G. W. collected the samples for further sequencing. X. Z. displayed the PCR experiment. X. Z. integrated the data and wrote the manuscript. P.-X. Q., Y.-J. L., and J. J. provided advices for this work. H.-H. Z. revised this manuscript. All authors approved the manuscript. X. Z., J.J. Q., H.-F. W., and Y.-G. W. were considered contributing equally to this work.

Funding

This study was funded by the National Natural Science Foundation of China (81800199), and the Natural Science Foundation of Zhejiang Province (LY21H080003).

Availability of data and materials All data generated or analyzed during this study are included in this published article. 


\section{Declarations}

\section{Ethics approval and consent to participate}

This study was approved by the ethical review committees of the First

Affiliated Hospital to Zhejiang University College of Medicine.

\section{Consent for publication}

Written informed consent was obtained from this patient.

\section{Competing interests}

The authors declare that they have no competing interests.

\section{Author details}

'Department of Hematology, The First Affiliated Hospital, Zhejiang University School of Medicine, \#79 Qingchun Rd, Hangzhou 310003, Zhejiang, China. ${ }^{2}$ Key Laboratory of Hematologic Malignancies, Diagnosis and Treatment, Hangzhou, Zhejiang, China. ${ }^{3}$ Zhejiang University Cancer Center, Zhejiang, Hangzhou, China. Institute of Hematology, Zhejiang University, Hangzhou, Zhejiang, People's Republic of China. ${ }^{5}$ Zhejiang Laboratory for Systems \& Precision Medicine, Zhejiang University Medical Center, 1369 West Wenyi Road, Hangzhou 311121, China.

Received: 5 January 2021 Accepted: 20 April 2021

Published online: 01 May 2021

\section{References}

1. DiNardo CD, Pratz K, Pullarkat V, Jonas BA, Arellano M, Becker PS, et al. Venetoclax combined with decitabine or azacitidine in treatment-naive, elderly patients with acute myeloid leukemia. Blood. 2019;133(1):7-17. https://doi.org/10.1182/blood-2018-08-868752.

2. DiNardo CD, Jonas BA, Pullarkat V, Thirman MJ, Garcia JS, Wei AH, et al. Azacitidine and venetoclax in previously untreated acute myeloid leukemia. N Engl J Med. 2020;383(7):617-29. https://doi.org/10.1056/NEJMoa2012971.

3. Pollyea DA, Pratz K, Letai A, Jonas BA, Wei AH, Pullarkat V, et al. Venetoclax with azacitidine or decitabine in patients with newly diagnosed acute myeloid leukemia: long term follow-up from a phase 1b study. Am J Hematol. 2021;96(2):208-17.

4. DiNardo CD, Tiong IS, Quaglieri A, MacRaild S, Loghavi S, Brown FC, et al. Molecular patterns of response and treatment failure after frontline venetoclax combinations in older patients with AML. Blood. 2020;135(11): 791-803. https://doi.org/10.1182/blood.2019003988.

5. Tausch E, Close W, Dolnik A, Bloehdorn J, Chyla B, Bullinger L, et al. Venetoclax resistance and acquired BCL2 mutations in chronic lymphocytic leukemia. Haematologica. 2019;104(9)::434-7. https://doi.org/10.3324/haematol.2019.222588.

6. Blombery P, Thompson ER, Nguyen T, Birkinshaw RW, Gong JN, Chen X, et al. Multiple BCL2 mutations cooccurring with Gly101Val emerge in chronic lymphocytic leukemia progression on venetoclax. Blood. 2020; 135(10):773-7. https://doi.org/10.1182/blood.2019004205.

7. Blombery P, Anderson MA, Gong JN, Thijssen R, Birkinshaw RW, Thompson ER, et al. Acquisition of the recurrent Gly101Val mutation in BCL2 confers resistance to venetoclax in patients with progressive chronic lymphocytic leukemia. Cancer Discov. 2019;9(3):342-53. https://doi.org/10.1158/2159-8290.CD-18-1119.

8. Blombery P, Birkinshaw RW, Nguyen T, Gong JN, Thompson ER, Xu Z, et al. Characterization of a novel venetoclax resistance mutation (BCL2 Phe104lle) observed in follicular lymphoma. Br J Haematol. 2019;186(6):e188-91. https://doi.org/10.1111/bjh.16069.

9. Birkinshaw RW, Gong JN, Luo CS, Lio D, White CA, Anderson MA, et al. Structures of BCL-2 in complex with venetoclax reveal the molecular basis of resistance mutations. Nat Commun. 2019;10(1):2385. https//doi.org/10.1038/s41467-019-10363-1.

10. Nechiporuk T, Kurtz SE, Nikolova O, Liu T, Jones CL, D'Alessandro A, et al. The TP53 apoptotic network is a primary mediator of resistance to BCL2 inhibition in AML cells. Cancer Discov. 2019;9(7):910-25. https//doi.org/10.1158/2159-8290.CD-19-0125.

11. Cancer Genome Atlas Research N, Ley TJ, Miller C, Ding L, Raphael BJ, Mungall AJ, et al. Genomic and epigenomic landscapes of adult de novo acute myeloid leukemia. N Engl J Med. 2013;368:2059-74.

12. Coutre $S$, Choi M, Furman RR, Eradat $H$, Heffner $L$, Jones JA, et al. Venetoclax for patients with chronic lymphocytic leukemia who progressed during or after idelalisib therapy. Blood. 2018;131(15):1704-11. https//doi.org/10.1182/blood-2017-06-788133.

\section{Publisher's Note}

Springer Nature remains neutral with regard to jurisdictional claims in published maps and institutional affiliations.

Ready to submit your research? Choose BMC and benefit from:

- fast, convenient online submission

- thorough peer review by experienced researchers in your field

- rapid publication on acceptance

- support for research data, including large and complex data types

- gold Open Access which fosters wider collaboration and increased citations

- maximum visibility for your research: over $100 \mathrm{M}$ website views per year

At BMC, research is always in progress.

Learn more biomedcentral.com/submissions 